\title{
Tensile Properties of Contractile and Synthetic Vascular Smooth Muscle Cells*
}

\author{
Hiroshi MIYAZAKI**, Yoshitaka HASEGAWA*** \\ and Kozaburo HAYASHI**
}

\begin{abstract}
Tensile properties of vascular smooth muscle cells (VSMCs) of synthetic and contractile phenotypes were determined using a newly developed tensile test system. Synthetic and contractile VSMCs were isolated from the rabbit thoracic aorta with an explant and an enzymatic digestion method, respectively. Each cell floated in Hanks' balanced salt solution of $37^{\circ} \mathrm{C}$ was attached to the fine tips of a pair of micropipettes with a cell adhesive and, then, stretched at the rate of $6 \mu \mathrm{m} / \mathrm{sec}$ by moving one of the micropipettes with a linear actuator. Load applied to the cell was measured with a cantilever-type load cell; its elongation was determined from the distance between the micropipette tips using a video dimension analyzer. The synthetic and contractile VSMCs were not broken even at the tensile force of $2.4 \mu \mathrm{N}$ and $3.4 \mu \mathrm{N}$, respectively. Their stiffness was significantly higher in contractile phenotype $(0.17 \mathrm{~N} / \mathrm{m})$ than in synthetic one $(0.09 \mathrm{~N} / \mathrm{m})$. The different tensile properties between synthetic and contractile cells are attributable to the differences in cytoskeletal structures and contractile apparatus.
\end{abstract}

Key Words : Biomechanics, Measurement, Vascular Smooth Muscle Cell, Phenotype, Tensile Testing, Stiffness, Cellular Mechanics

\section{Introduction}

It is well known that vascular smooth muscle cells (VSMCs) have two phenotypes, contractile and synthetic, and their structures and functions are quite different from each other ${ }^{(1)-(4)}$. These cells play important roles not only in vascular tone but also in arterial remodeling, repair, and growth ${ }^{(1)}$. The vast majority of VSMCs in the media of normal adult arteries are of contractile phenotype in quiescent state. They have contractile apparatus composing of actin and myosin filaments, and few synthetic organelles including rough endoplasmic reticulum, Golgi apparatus, and free ribosomes ${ }^{(1)-(5)}$. Contractile VSMCs maintain vascular tone and actively regulate blood pressure

* Received 31st May, 2002 (No. 02-4093)

** Division of Mechanical Science, Department of Systems and Human Science, Graduate School of Engineering Science, Osaka University, Toyonaka, Osaka 560-8531, Japan. E-mail : oboe@me.es.osaka-u.ac.jp (Miyazaki)

*** Advanced Technology R\&D Center, Mitsubishi Electric Corporation, Amagasaki, Hyogo 661-8661, Japan through contraction and relaxation. Their contraction changes not only blood pressure but also the mechanical properties of arterial wall ${ }^{(6),(7)}$. On one hand, synthetic VSMCs are present in developing and regenerating arteries ${ }^{(1)-(4)}$. Their structures and functions are similar to those of fibroblasts; they actively proliferate, and have few contractile filaments but large amount of synthetic organelles associated with production of both intracellular components and extracellular matrix such as collagen, elastin, and proteoglycans ${ }^{(1)-(4),(8)}$.

VSMCs change their phenotype in response to mechanical and hormonal stimuli, and also to environmental changes such as alterations of surrounding extracellular matrix components ${ }^{(1)-(4),(9)-(13)}$. This phenotypic modulation of VSMCs is one of the key events in the two major vascular diseases: atherosclerosis and hypertension ${ }^{(1),(3),(12)}$. At an early stage of atherosclerosis, VSMCs migrate from the wall media to the intima, and modulate their phenotype from contractile to synthetic ${ }^{(1),(3),(12),(14)}$. Then, they proliferate, synthesize and secrete extracellular matrix components, and promote the development of atherosclerotic lesions. On one hand, hypertension induces 
arterial wall thickening accompanied with hyperplasia of $\mathrm{VSMCS}^{(1),(15)}$ and change in connective tissue content ${ }^{(1),(16)}$

Intracellular structures, especially cytoskeletal systems, affect the mechanical properties of cells including vascular endothelial cells ${ }^{(17),(18)}$, fibroblasts $^{(19)}$, and neutrophils ${ }^{(20)}$. Therefore, the phenotypic modulation should be reflected to the mechanical properties of VSMCs, because it markedly changes their intracellular structures ${ }^{(1)-(4),(10)}$. Since the functions of cells are closely related to their morphology, components, and intracellular structures, measurement of mechanical properties of VSMCs should provide very important information of the process and mechanisms of phenotypic modulation. It is therefore very important to know the mechanical properties of both phenotypes of VSMCs for understanding not only the physiology of VSMCs themselves but also the basic physiology, pathology, and mechanics of arterial wall. However, little is known about the mechanical properties of both phenotypes of VSMCs.

Various methods and techniques have been applied to the determination of the mechanical properties of cells ${ }^{(17)-(24)}$, many of which can be used for local properties in a cell. Such local properties provide information of the mechanical linkage among cell membrane, cytoskeleton, organelles, and nucleus in restricted regions inside a cell. However, to understand cell functions and physiology, it is very important to know the structure and properties of a whole cell. Since cells are structurally and mechanically inhomogeneous ${ }^{(19),(22)}$, local properties do not represent their whole properties. Therefore, the measurement of the mechanical properties of a whole cell is not only basically important but also prerequisite to understand the intracellular structures and interactions among intracellular components throughout the cell. For the study of basic mechanics of a whole cells, such a simple test as uniaxial tensile testing is useful and preferable. However, few studies have been done on the tensile properties of cells ${ }^{(21),(23),(25)}$, partly because the tensile testing of a whole cell is hard to do mainly due to the difficulty in cell gripping. Glerum et al. ${ }^{(21)}$ determined the tensile properties of single smooth muscle cells obtained from the urinary bladder and uterus. They gripped a cell by knotting each end of the cell around each tip of a pair of micropipettes. However, their gripping technique can be applied only to relatively long and stiff cells. Matsumoto et al. ${ }^{(25)}$ studied the tensile properties of rat VSMCs freshly isolated with an enzymatic digestion method and of cultured bovine VSMCs. They aspirated both ends of a cell into a pair of micropipettes which were coated with a cell adhesive. Their gripping technique was essentially similar to our previously reported method $^{(23)}$. However, they could determine the properties of only the central part of a cell, because its fairly large portions were aspirated into the two micropipettes.

Recently, we have developed a micro tensile test system which can be used for many kinds of cells and fine biological fibers, and have been using it to determine the mechanical properties of fibroblasts ${ }^{(23)}$ and collagen fibers ${ }^{(26)}$. In this system, a cell is attached to the fine tips of a pair of micropipettes using a cell adhesive, which makes it possible to grip a small and spherical cell. The properties of a whole cell can be determined, since no portions are aspirated into the micropipettes. In the present study, the tensile properties of both synthetic and contractile phenotypes of VSMCs obtained from rabbit thoracic aortas were determined using the system to study the effect of phenotypic modulation.

\section{Materials and Methods}

\section{1 Preparation of vascular smooth muscle cells of synthetic phenotype}

All animal experiments were conducted under the Guideline for Animal Experiments, Graduate School of Engineering Science, Osaka University.

Synthetic phenotype of VSMCs were isolated from mature female Japanese white rabbits weighing approximately $3 \mathrm{~kg}$ with an explant $\operatorname{method}^{(27)}$. The thoracic aorta was exposed under anesthesia induced by the injection of pentobarbital sodium into the auricular vein. Immediately after sacrifice with an excess of pentobarbital sodium, the aorta between the ligamentum arteriosum and the third intercostal arteries was aseptically excised, and immersed in $\mathrm{Ca}^{2+-}$ and $\mathrm{Mg}^{2+-}$ free Hanks' balanced salt solution $(\operatorname{HBSS}(-))$. The composition of the $\operatorname{HBSS}(-)$ was: $136.8 \mathrm{NaCl}, 5.3 \mathrm{KCl}, 0.3 \mathrm{Na}_{2} \mathrm{HPO}_{4} \cdot 12 \mathrm{H}_{2} \mathrm{O}, 0.4$ $\mathrm{KH}_{2} \mathrm{PO}_{4}, 4.2 \quad \mathrm{NaHCO}_{3}$, and 5.6 dextrose (in millimolar). The aortic segment was cut opened lengthwise and put in a culture dish with the intimal side up. After the endothelium was removed by gentle scrape with a spatula, a thin medial layer at the intimal side was carefully peeled off with a pair of forceps. The detached media was then cut into small rectangular pieces each having the length and width of approximately 1 and $0.5 \mathrm{~mm}$, respectively, with a pair of surgical blades, and immersed in a small amount of Dulbecco's Modified Eagle Medium (DMEM, Life Technologies Inc., Rockville, MD, USA) supplemented with $10 \%$ fetal calf serum (FCS, HyClone, UT) , 10 mM HEPES, $100 \mathrm{U} / \mathrm{ml}$ penicillin, and $100 \mu \mathrm{g} / \mathrm{ml}$ streptomycin (complete DMEM). These pieces were 
placed on the bottom of a tissue culture dish, and incubated at $37^{\circ} \mathrm{C}$ for $30 \mathrm{~min}$. Then, they were cultured in complete DMEM at $37^{\circ} \mathrm{C}$. Approximately 6 days after, VSMCs started migrating from the explants, and became confluent at approximately 2 weeks. The cells were harvested with phosphatebuffered saline (PBS) containing 0.25\% trypsin, and subcultured once in the complete DMEM in a tissue culture flask. After confluence was reached, they were harvested using the trypsin solution, and suspended in a $\operatorname{HBSS}(+)$. The composition of $\operatorname{HBSS}(+)$ was:

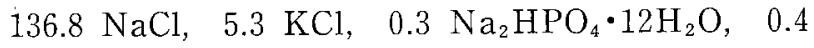
$\mathrm{KH}_{2} \mathrm{PO}_{4}, \quad 0.5 \mathrm{MgCl}_{2} \cdot 6 \mathrm{H}_{2} \mathrm{O}, \quad 0.4 \mathrm{MgSO}_{4} \cdot 7 \mathrm{H}_{2} \mathrm{O}, \quad 1.3$ $\mathrm{CaCl}_{2}, 4.2 \mathrm{NaHCO}_{3}$, and 5.6 dextrose (in millimolar).

2. 2 Preparation of vascular smooth muscle cells of contractile phenotype

Contractile phenotype of VSMCs were isolated with an enzymatic digestion method. Small medial pieces obtained from the rabbit thoracic aorta with the procedures mentioned above were put in a tissue culture flask with $10 \mathrm{ml}$ of DMEM containing nonessential amino acid (Life Technologies Inc., Rockville, MD), $10 \mathrm{mg}$ collagenase (Nitta Gelatin Inc., Osaka, Japan), and 0.5 mg elastase (Sigma, St. Louis, $\mathrm{MO})$. For digestion, they were gently shaken at a rate of $1 \mathrm{cycle} / \mathrm{sec}$ at $37^{\circ} \mathrm{C}$ for about $3 \mathrm{~h}$. Isolated VSMCs were washed and cultured on collagen-coated coverslips in the complete DMEM for 3 days. The cells attached to the coverslip surfaces were harvested with PBS containing 0.25\% trypsin, and washed with the complete DMEM. Then, they were suspended in a $\operatorname{HBSS}(+)$.

\section{3 Fluorescent microscopy}

To confirm the phenotypes of VSMCs, the expression of smooth muscle myosin heavy chain (SMMHC) was examined using an immunofluorescent staining technique, which is a modification of the method used by Halayko et al. ${ }^{(28)}$ Briefly, several drops of cell suspension were placed on glass coverslips coated with poly-L-lysine (Sigma, St. Louis, MO). After $5 \mathrm{~min}$, the coverslips were rinsed with PBS, and attached cells were fixed with PBS containing 3.7\% formaldehyde for $10 \mathrm{~min}$, followed by rinsing with PBS. The cells were soaked in PBS containing $0.1 \%$ Triton $\mathrm{X}-100$ for $30 \mathrm{~min}$. Then, they were rinsed with PBS and incubated with antibody to SM-1 and SM-2 isoforms of SM-MHC (Sigma) in PBS containing $5 \%$ bovine serum albumin (BSA, Sigma) for $1 \mathrm{~h}$. They were rinsed in PBS again, and incubated with FITC-conjugated anti-mouse IgG (Sigma) diluted with PBS containing 5\% BSA for $45 \mathrm{~min}$, and finally rinsed with PBS. All of these procedures were carried out at room temperature.

Thus stained cells were observed with a confocal laser scanner (CSU10, Yokogawa Electric Co., Tokyo, Japan) combined with an inverted microscope (IX-70, Olympus, Tokyo, Japan) which is installed in the tensile test system used for the present study. An argon laser (5490ASL, Ion Laser Technology, Salt Lake City, UT) having the wavelength of $488 \mathrm{~nm}$ was used for the excitation of FITC, and photographs of the cells were taken on $35-\mathrm{mm}$ color film (Fujifilm, Tokyo, Japan).

Actin filaments were also observed with the fluorescent microscopy. VSMCs spread out on coverslips were rinsed with PBS, and they were fixed with PBS including $3.7 \%$ formaldehyde for $10 \mathrm{~min}$, followed by rinsing with physiological saline solution. The cells were treated with $0.1 \%$ Saponin (Wako Pure Chemical, Osaka, Japan) in physiological saline solution for $20 \mathrm{~min}$. Then, they were rinsed with physiological saline solution and incubated with $12.5 \mathrm{mg} / \mathrm{l}$ of FITCphalloidin (Sigma) in PBS containing 1\% dimethylsulfoxide (Wako Pure Chemical) for $20 \mathrm{~min}$. They were finally rinsed with PBS. All the procedures were carried out at room temperature. Thus treated cells were observed with the confocal laser scanner mentioned above.

\section{4 Contraction test with norepinephrine}

Contractile response of VSMCs to norepinephrine was observed with phase contrast microscopy. The cells which were cultured on the surfaces of tissue culture flask or of the coverslips described above were detached from their substrates using $0.25 \%$ trypsin solution, and suspended to $10^{4}$ cells $/ \mathrm{ml}$ in $\operatorname{HBSS}(+)$. One $\mathrm{ml}$ of the cell suspension was added to $11 \mathrm{ml}$ of $\mathrm{HBSS}(+)$ previously bubbled with $100 \% \mathrm{O}_{2}$ at $37^{\circ} \mathrm{C}$, and it was put in a thermostatic test chamber of the tensile test system. The image of cells was recorded on a videotape via the microscope and a CCD camera. Then, norepinephrine (Sankyo Co., Tokyo, Japan) was added to the test chamber until a final concentration of $10^{-5} \mathrm{M}$ was reached. Morphological changes of the cells were videotaped for $15 \mathrm{~min}$. Contractile response was examined on 10 cells each from the cell populations obtained with the enzymatic digestion and the explant method.

\subsection{Tensile testing}

Tensile tester used for the present study was essentially similar to that reported previously ${ }^{(23)}$. It consists of a thermostatic test chamber, an inverted fluorescence microscope, two micromanipulators, a direct drive linear actuator, a cantilever-type load cell, and a video dimension analyzer (VDA). The present system has been newly incorporated with the above-mentioned confocal laser scanner for the observation of intracellular structure and a laser displacement meter (LC-2440, Keyence, Osaka, Japan) for the 


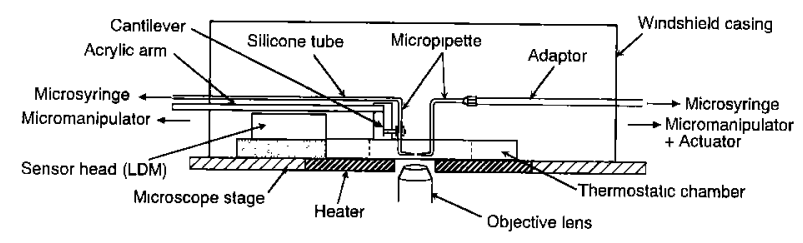

Fig. 1 Main part of the tensile test system for cells. LDM $=$ Laser displacement meter

determination of load applied to a cell. Main part of the system is shown in Fig. 1. The cantilever of the load cell was made of a rectangular stainless steel plate $(30 \mu \mathrm{m}$ thick), to which a laser beam reflector was attached. The load was determined from the deflection of the cantilever measured with the laser displacement meter. The cantilever was attached to an acrylic arm which is connected to a piezoelectric micromanipulator (MP-300, Narishige, Tokyo, Japan).

Test procedures have been previously described in detail ${ }^{(23)}$. Briefly, a pair of double-L-shaped micropipettes were fabricated from glass capillary tubes ( $1 \mathrm{~mm}$ outer diameter and $0.56 \mathrm{~mm}$ inner diameter ; Narishige). Their fine tips (outer diameter $=20-40$ $\mu \mathrm{m}$; inner diameter $=3-5 \mu \mathrm{m}$ ) used for gripping a cell were coated with a cell adhesive, Cell-Tak (Collaborative Biomedical Products, Bedford, MA). One of the micropipettes was attached to the cantilever, and its wide end was connected to a microsyringe via a silicone tube (Fig. 1). The other one was connected to another micromanipulator (MHW-103, Narishige) which can be moved by the linear actuator (UCM410-5C, Oriental Motor, Tokyo, Japan), and its wide end was connected to a microsyringe via a Teflon tube. These micropipettes were filled with HBSS. A cell floated in HBSS of $37^{\circ} \mathrm{C}$ in the test chamber was attached to the fine tip of one of the micropipettes which was connected to the load cell, by applying very low negative pressure produced by the microsyringe. The pressure application was continued for 15 to $20 \mathrm{~min}$ until the cell was firmly bonded to the micropipette tip. The silicone tube between the micropipette and the microsyringe was cut gently to return the pressure to atmospheric pressure and also to apply testing load directly to the load cell. Then, the fine tip of the other micropipette was attached to the opposite side of the cell in a fashion similar to that mentioned above. After returning internal pressure in the micropipette to atmospheric pressure, the cell was stretched at a rate of $6 \mu \mathrm{m} / \mathrm{sec}$. During tensile testing, load was determined from the deflection of the cantilever, while cell elongation was measured from the distance between the edges of both micropipette tips using the VDA (C3160, Hamamatsu Photonics, Hama-

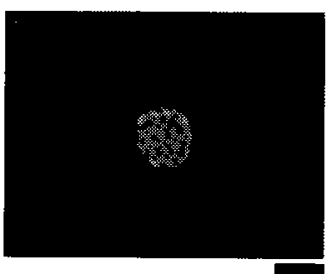

(a)

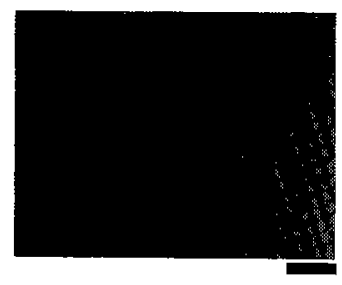

(b)
Fig. 2 Examples of the phase contrast and confocal laser scanning micrographs of SM-MHC stained VSMCs. (a) a cell isolated with enzymatic digestion method; (b) a cell with explant method. Bar, $20 \mu \mathrm{m}$

matsu, Japan) via the microscope and a CCD camera (WV-BP310, Matsushita Communication Industrial Co., Yokohama, Japan). After tensile testing, the load cell was calibrated using a reference glass microneedle having a known spring constant which was determined by a cross-calibration method. The accuracy of force measurement was $\pm 0.05 \mu \mathrm{N}$, and the resolution of displacement measurement was. $0.24 \mu \mathrm{m}$.

\section{Results}

Examples of confocal laser scanning micrographs of SM-1/SM-2-stained VSMCs are shown in Fig. 2. Abundant SM-1/SM-2 SM-MHC isoforms were observed in the cells which were isolated with the enzymatic digestion method (Fig. 2(a)). On the other hand, almost no SM-1/SM-2 were observed in the cells obtained from the explants (Fig. 2(b)). Seventy percent of the cells isolated with the digestion method contracted in response to $10^{-5} \mathrm{M}$ norepinephrine, and formed remarkable membranous evaginations on the cell surface (Fig. 3(a) right). These evaginations appeared within $20 \mathrm{~s}$ after the administration of norepinephrine, and changed size and positions for up to 15 min. However, the cells obtained from the explants did not respond much to norepinephrine (Fig. 3(b)). These results indicate that the cells isolated with the enzymatic digestion method were of contractile phenotype, and that those obtained from the explant method were of synthetic one.

Figure 4 shows examples of confocal laser scanning micrographs of actin filaments in VSMCs adhered to coverslips. Actin bundles were much thicker in contractile cells than in synthetic ones.

The shape of the cells isolated with the abovementioned methods were almost spherical before tensile tests ; averaged diameter of non-loaded synthetic and contractile cells were $32.3 \pm 7.4 \mu \mathrm{m}$ and $31.0 \pm 3.9$ $\mu \mathrm{m}$ (mean \pm S.D. ; $n=6$ ), respectively. There was no significant difference in the diameter between the two phenotypes of cells.

During tensile testing, the shape of most cells was 

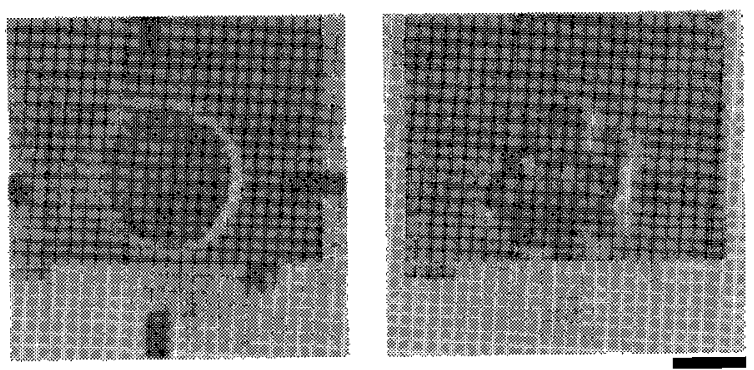

(a)
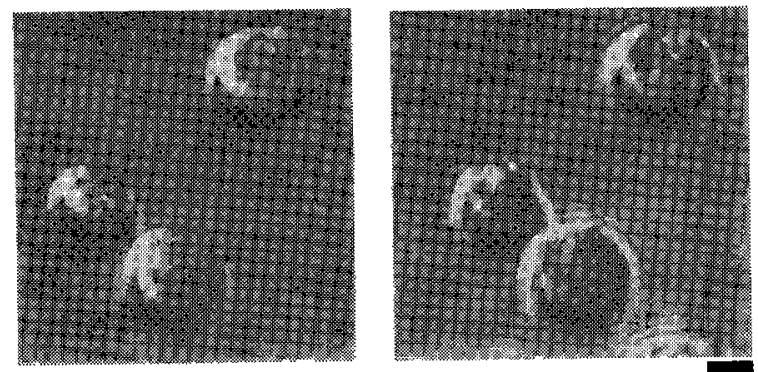

(b)

Fig. 3 Examples of the phase contrast micrographs of VSMCs before (left) and after (right) the administration of norepinephrine. (a) enzymatic digestion method; (b) explant method. Bar, $10 \mu \mathrm{m}$.

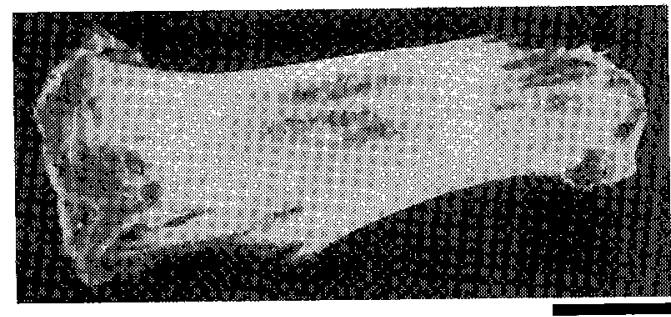

(a)

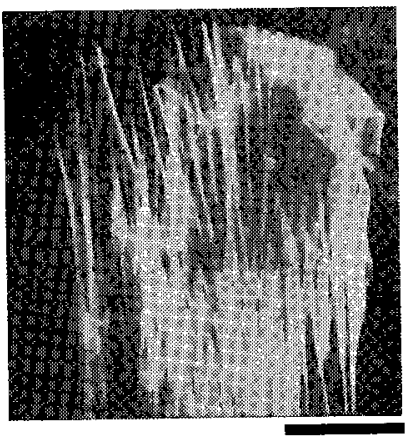

(b)

Fig. 4 Examples of the confocal laser scanning micro graphs showing actin filaments in VSMCs. (a) contractile VSMC; (b) synthetic VSMC. Bar, 50 $\mu \mathrm{m}$

irregular (Fig. 5), which may reflect the inhomogeneous structures inside the cells. Load-elongation relations obtained from six VSMCs of each phenotype are shown in Fig. 6. All the cells showed essentially
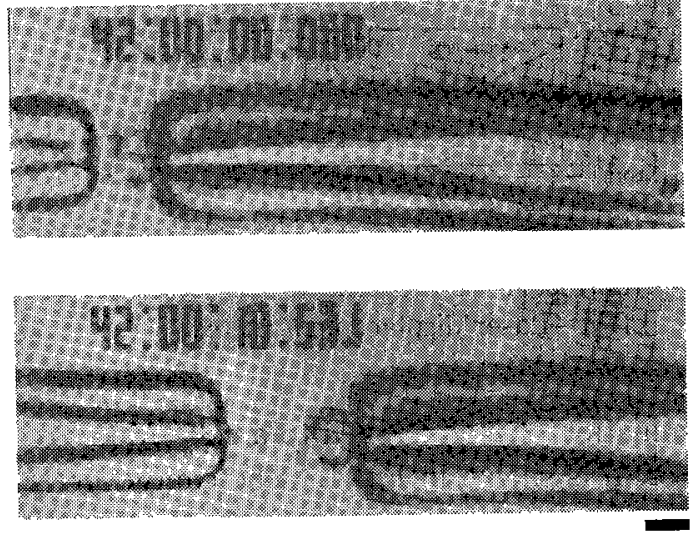

Fig. 5 A contractile VSMC during tensile testing. Bar, $20 \mu \mathrm{m}$

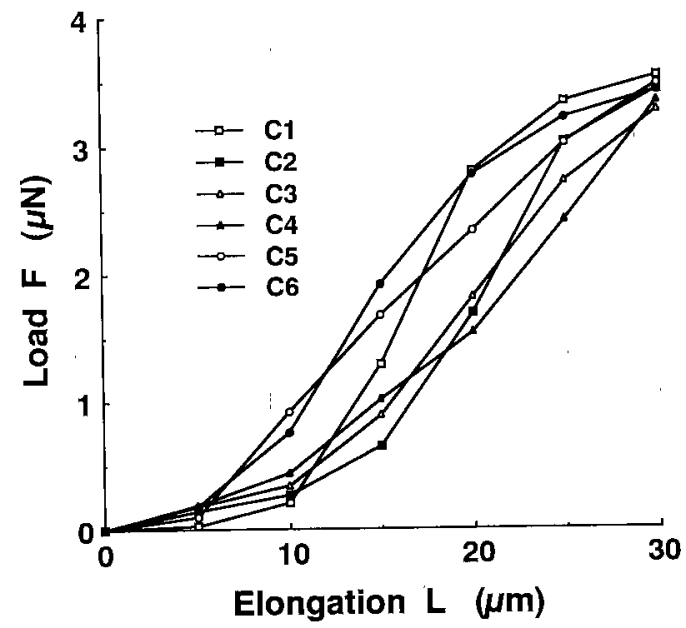

(a)

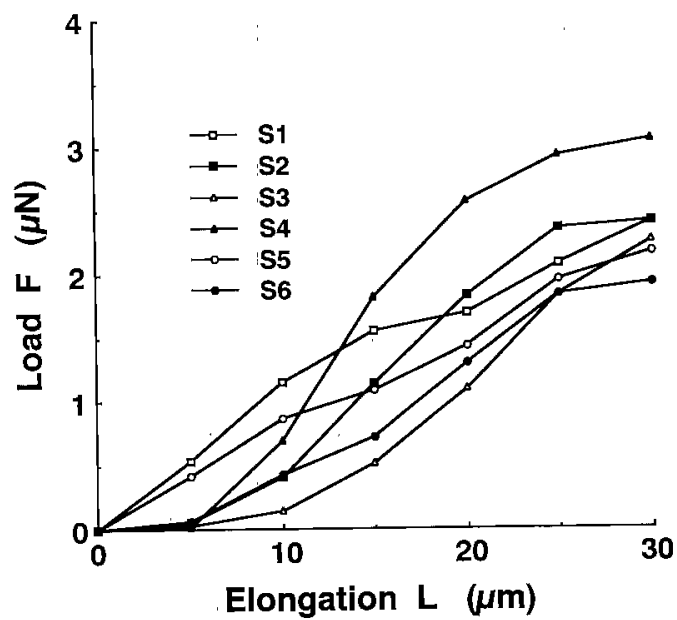

(b)

Fig. 6 Load vs. elongation of VSMCs. Symbols indicate individual cells. (a ) contractile VSMC ; (b) synthetic VSMC

similar load-elongation relations, although they were variable. We could not determine the maximum failure load, because the cells were all detached from one of the micropipette tips before breaking. Figure 


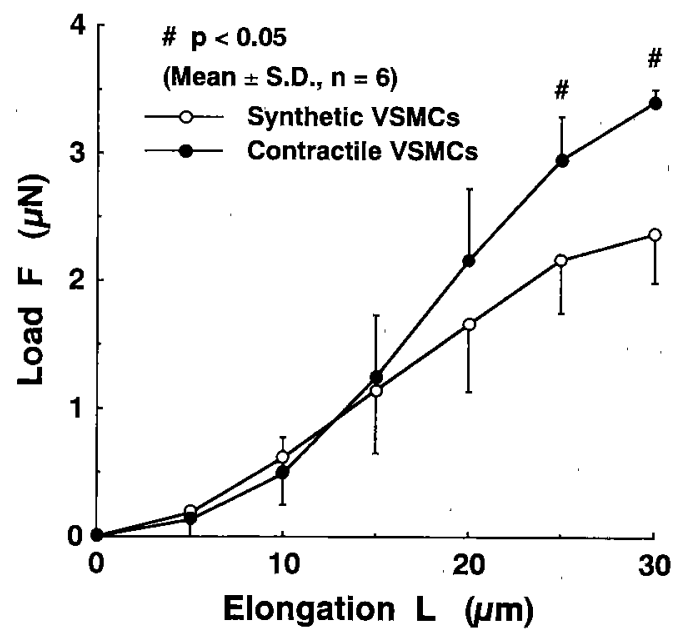

Fig. 7 Averaged load vs. elongation relations of the two phenotypes of VSMCs

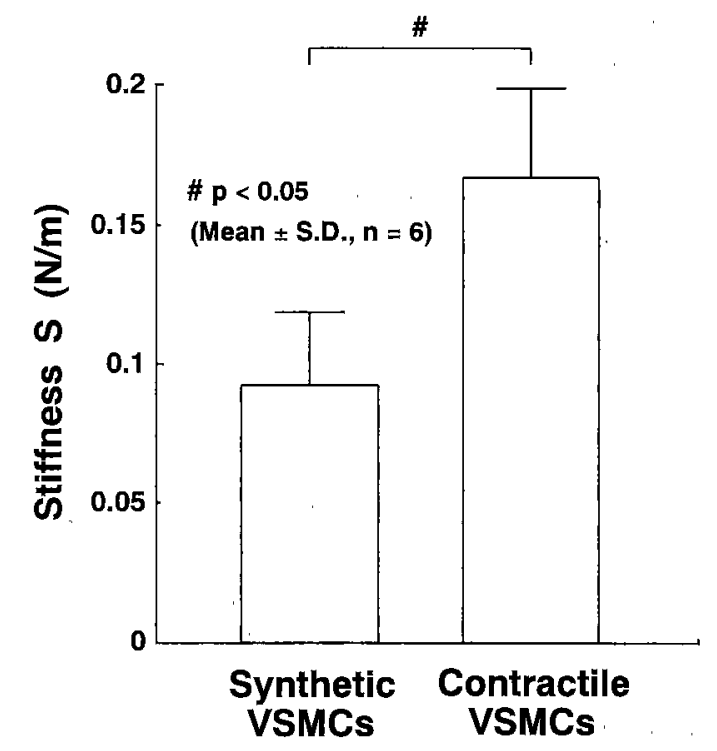

Fig. 8 Stiffness of synthetic and contractile VSMCs. The stiffness was defined by slope of load-elongation curve between 10 and $25 \mu \mathrm{m}$ elongation

7 shows the averaged load-elongation relations. Both phenotypes of cells showed almost the same loadelongation relations in small elongation ranges. However, tensile load was higher in contractile VSMCs than in synthetic ones at larger elongation than $20 \mu \mathrm{m}$; there were significant differences at 25 and $30 \mu \mathrm{m}$ elongation. Synthetic and contractile cells did not break up to the load of $2.4 \mu \mathrm{N}$ and $3.4 \mu \mathrm{N}$, respectively.

The stiffness of VSMCs was significantly higher in the contractile phenotype $(0.17 \pm 0.032 \mathrm{~N} / \mathrm{m})$ than in the synthetic one $(0.09 \pm 0.027 \mathrm{~N} / \mathrm{m})$ (Fig. 8), where the stiffness was defined by the slope of the nearly linear portion of each load-elongation curve between 10 and $25 \mu \mathrm{m}$ elongation.

\section{Discussion}

We studied the effect of phenotypic modulation on the mechanical properties of VSMCs using rabbit aortic SMCs prepared with two different methods : an explant and an enzymatic digestion methods. The cells migrated from explants actively proliferated and reached confluence after about 2 weeks, and were then subcultured once. On one hand, the cells isolated with the enzymatic digestion method were cultured only for 3 days before tensile testing. It is known that SMCs migrated from explants and then subcultured have features of synthetic phenotype ${ }^{(2),(27)}$. It is also known that enzyme-dispersed VSMCs in primary culture retain contractile state for the first few days, and that they change their phenotype to synthetic after 6 to 8 days $^{(10)}$. Therefore, it was expected that the cells obtained with the explant and enzymatic digestion methods in the present study were synthetic and contractile VSMCs, respectively, although we did not observe their intracellular structures with electron microscopy. To confirm the phenotypes of the cells used for the present study, we observed the expression of SM-MHC isoforms SM-1 and SM-2 and also studied the response of isolated cells to norepinephrine. Isoforms SM-1 and SM-2, particularly SM-2, are predominantly expressed in differentiated, contractile SMCs, whereas dedifferentiated SMCs predominantly express nonmuscle $\mathrm{MHC}^{(11),(12),(28)}$. In the present study, confocal laser scanning microscopy showed abundant SM-1/SM-2 in the cells which were harvested with the enzymatic digestion method, but almost no SM-1/SM-2 in the cells obtained from the explants (Fig. 2). In addition, 70\% of the cells isolated with the digestion method contracted and developed membranous evaginations in response to norepinephrine; however, the cells obtained from the explants almost did not respond to norepinephrine (Fig. 3). From these results, we can say that the cells isolated with the enzymatic digestion method were of contractile phenotype, and those obtained with the explant method were of synthetic one. Ives et al. ${ }^{(29)}$ isolated SMCs from the rabbit thoracic aorta with an enzymatic digestion method and examined their response to agonists. They reported that the cells cultured for 1 to 5 days after dissociation contracted in response to agonists, and that less extended cells developed cytoplasmic evaginations. Morphological change of VSMCs observed in the present study was similar to that observed in their study. They also reported that not all the cells responded to agonists, and that the proportion of cells contracting in response to agonists was greater after $24 \mathrm{~h}$ culture than immediately after dissociation. From this result, they suggested that 
hormone binding ability and/or contractile function damaged during dissociation were restored by culture ${ }^{(29)}$. In the present study, $30 \%$ of the cells isolated with the digestion method did not show visible morphological changes. It may be attributable to some damage of cells induced by impurities in the enzyme solution during dissociation or to the heterogeneity of SMCs in the arterial wall ${ }^{(12)}$.

Simple tensile testing is very useful for the study of basic mechanical properties of a whole cell. To our knowledge, there have been only a few studies on the tensile properties of cells $^{(21),(23),(25)}$. Glerum et al. ${ }^{(21)}$ determined the tensile properties of SMCs harvested from the pig urinary bladder and human uterus, and reported that the maximum load was as high as 70 to $100 \mu \mathrm{N}$. Miyazaki et al. ${ }^{(23)}$ determined the tensile properties of fibroblasts obtained from the rabbit patellar tendon, and showed that their maximum load and elongation at failure were approximately $0.9 \mu \mathrm{N}$ and $86 \mu \mathrm{m}$, respectively. Recently, Matsumoto et a1. ${ }^{(25)}$ studied the tensile properties of rat aortic SMCs freshly isolated with an enzymatic digestion method and those of cultured bovine aortic SMCs. They reported that elastic modulus was significantly higher in the fresh rat cells than in the cultured bovine cells. However, they did not identify the phenotype of the cells, and the sources of the two cell populations were different. The present experiment is the first one which precisely determined the tensile force-elongation relations of two phenotypes of VSMCs for the study of the effect of phenotypic modulation on the mechanical properties.

We could not determine the maximum load at failure, because all the cells were detached from one of the micropipette tips before cell breaking. In a few cells, load at $30 \mu \mathrm{m}$ elongation were almost the same as those at $25 \mu \mathrm{m}$ elongation (Fig. 6) ; these cells may have started detaching from one of the micropipette tips at $25 \mu \mathrm{m}$ elongation. Thus, we obtained the stiffness of each cell from the slope of the nearly linear portion of the load-elongation curve between 10 and $25 \mu \mathrm{m}$ elongation.

The stiffness was much higher in contractile VSMCs $(0.17 \mathrm{~N} / \mathrm{m})$ than in synthetic ones $(0.09 \mathrm{~N} / \mathrm{m})$ (Fig. 8), which indicates that mechanical properties are changed by phenotypic modulation. As shown in Fig. 4, actin bundles were much thicker in contractile VSMCs than in synthetic ones. Contractile SMCs have a contractile apparatus composed of actin and myosin filaments, and a cytoskeleton comprising of actin filaments and intermediate filaments ${ }^{(5)}$. SMCs express several actin isoforms including smooth muscle $\alpha$-actin, nonmuscle $\beta$-actin, and nonmuscle $\gamma$ $\operatorname{actin}^{(3)}$. Smooth muscle and nonmuscle actin isoforms are found in the contractile apparatus and cytoskeleton, respectively ${ }^{(5)}$. The most abundant actin isoform in contractile SMCs is smooth muscle $\alpha^{-}$ actin. However, the amounts of nonmuscle $\beta^{-}$and $\gamma^{-}$ actin are increased, and inversely the expression of smooth muscle $\alpha$-actin is suppressed by phenotypic modulation $^{(4),(30)}$. Nonmuscle $\beta$-actin is a predominant isoform in synthetic $\mathrm{VSMCs}^{(30)}$. Because we used FITC-phalloidin to stain actin filaments, both smooth muscle actin in contractile apparatus and cytoskeletal actin should have been stained (Fig. 4). In addition, SM-MHC, which is one of the components of contractile apparatus, was abundant only in the VSMCs treated with the digestion method (Fig. 2). These results imply that the thicker actin bundles observed in the contractile cells (Fig. 4(a)) are of contractile apparatus. The contractile apparatus should contribute much to the tensile properties of contractile VSMCs, because contractile apparatus, cytoskeleton, and cell membrane are coupled by dense bodies and dense plaques, and the mechanical coupling transmits the tension generated by contractile apparatus throughout the cell and also to extracellular matrix ${ }^{(5),(31)}$. Load $^{-}$ elongation relations obtained from contractile and synthetic VSMCs were almost identical in small elongation ranges; however, tensile load was higher in contractile cells than synthetic ones at larger elongation than $20 \mu \mathrm{m}$ (Fig. 7). It is presumed that the loadelongation relation of contractile VSMCs was affected by the contractile apparatus in the large elongation range. Cytoskeletal systems such as intermediate filaments and nonmuscle actin filaments may also affect the load-elongation relations of both phenotypes of VSMCs, because the components of cytoskeletal systems are changed by phenotypic modulation ${ }^{(28)}$. We observed that the treatment of fibroblasts with cytochalasin B dramatically decreased their tensile stiffness and strength (unpublished result).

Glerum et al. ${ }^{(21)}$ reported that the maximum tensile load of smooth muscle cells obtained from the pig urinary bladder and human uterus were as high as 70 to $100 \mu \mathrm{N}$. Considering their results, the load-elongation relations shown in Fig. 7 may demonstrate only a low load region. Tensile load at $25 \mu \mathrm{m}$ elongation was $3.0 \pm 0.3 \mu \mathrm{N}$ in contractile VSMCs (Fig. 7), which is rather small compared with the value derived from the graphs given by Glerum et al. ${ }^{(21)}$ They obtained the data from only a small portion of a cell, because large end portions of the cell body (20 to $30 \mu \mathrm{m}$ for each end) were used for gripping. On the other hand, a whole cell was stretched in the present study. This difference, source of cells, and animal species might be the reasons for the difference between the two results, especially in initial regions. 
Matsumoto et al. ${ }^{(25)}$ reported that elastic modulus was significantly higher in freshly isolated rat aortic SMCs than in cultured bovine aortic ones. In the present study, we did not calculate elastic modulus nor stress, because these parameters give no physical meaning due to very irregular cell shape during tensile testing (Fig.5). Instead, we evaluated the tensile property by stiffness. Although Matsumoto et al. did not identify the phenotype of their cells, their results seem to be similar to ours if it is assumed that the rat cells were contractile and the bovine cells were synthetic. However, they reported that the slope of force-elongation curves or stiffness was not significantly different between the two cells, which is different from our results showing significantly higher stiffness in contractile VSMCs than in synthetic ones (Fig. 8). The stiffness of their rat VSMCs $(0.036 \pm$ $0.008 \mathrm{~N} / \mathrm{m}, n=8$, mean $\pm \mathrm{SEM}$ ) was much smaller than that of our rabbit contractile VSMCs $(0.17 \pm$ $0.032 \mathrm{~N} / \mathrm{m}$ ). The reason for the difference between the two studies is unclear, but there are several explanations. In our study, the shape of both phenotypes of cells was spherical under no load condition, and there was no significant difference in the averaged diameter between them. In their study, the shapes of the freshly isolated rat cells and cultured bovine cells were elongated and spherical, respectively. They reported that the unloaded diameter of the rat cells and bovine cells were 6 to $13 \mu \mathrm{m}$ and 12 to $20 \mu \mathrm{m}$, respectively. In addition, because both ends of a cell were remained aspirated into a pair of micropipettes during tensile testing, some portion of the cell was not stretched and also suction pressure was applied to the cell during stretching. In our case, tensile load was almost completely applied to a cell during testing. The differences in cell shape and gripping methods are attributable to the difference in the results between the two studies.

Miyazaki et al. ${ }^{(23)}$ showed that the maximum load and elongation at failure of fibroblasts were approximately $0.9 \mu \mathrm{N}$ and $86 \mu \mathrm{m}$, respectively. Their stiffness was $0.015 \pm 0.006 \mathrm{~N} / \mathrm{m}$ if calculated from the loadelongation curves between 30 and $50 \mu \mathrm{m}$ elongation. VSMCs are much stiffer and stronger than fibroblasts even if they are of synthetic phenotype; the stiffness of synthetic VSMCs is more than 6 times as high as that of fibroblasts. These results suggest that the structure of synthetic VSMCs is much different from that of fibroblasts, although major functions of both cells are the synthesis and proliferation of extracellular matrix components.

In our previous study, fibroblasts were firmly attached to micropipette tips until fracture ${ }^{(23)}$. As stated above, however, VSMCs were detached from one of the micropipette tips during tensile testing; therefore, we could not determine the maximum load at failure. Bonding strength between cell membrane and Cell-Tak-coated micropipette tips was larger than the strength of fibroblasts but smaller than that of VSMCs. Although the differences in tensile properties between the two phenotypes of VSMCs and also between VSMCs and fibroblasts were clearly shown from the present results, it is suggested to improve the bonding strength between a cell and micropipette tip for further studies.

In the present study, spherical-shaped cells were used for tensile testing, because cells became round due to the trypsin treatment for the detachment of cells from culture dishes. This is suitable to compare the difference in mechanical properties between synthetic and contractile VSMCs, because it provides the same test condition for both cells. However, SMCs in the arterial wall are elongated and aligned almost in parallel to the wall circumferential direction. If elongated VSMCs are further stretched in the longitudinal direction, their load-elongation relations might be different from those of spherical cells, because the tension generated by contraction should be mainly directed to the longitudinal axis, and the orientation and conformation of contractile apparatus and cytoskeleton are arranged for this purpose. Tensile tests of elongated VSMCs should be done.

The shape of the load-elongation curves of two synthetic VSMCs was different from that of the other ones in a small elongation region (Fig. 6(b)). The synthetic cells adhered to the culture dish were elongated, which aligned actin filaments in parallel to the Iongitudinal axis of the cells (Fig. 4(b)). However, their shape became almost spherical after detachment, and we could not identify their original longitudinal axis. In tensile tests, the cells were stretched in random directions. Therefore, the anisotropic substructure of cells may have yielded such different shape of load-elongation curves among the cells, especially in a small elongation range.

The observation of conformational changes in contractile apparatus and cytoskeleton during tensile testing would be interesting for the detailed analysis of the function and mechanics of VSMCs. It should provide useful information not only of the contribution of contractile apparatus and individual cytoskeletal network systems to the mechanical properties of VSMCs but also of mechanotransduction in the response of VSMCs to mechanical stress.

In the present study, we determined the tensile properties of synthetic and contractile VSMCs obtained from the rabbit thoracic aorta, and studied the effects of phenotypic modulation on the mechani- 
cal properties. The stiffness of VSMCs was changed by phenotypic modulation; it was significantly higher in contractile phenotype than in synthetic one. The large differences in tensile properties between synthetic and contractile cells are attributable to the differences in cytoskeletal structures and contractile apparatus.

\section{Acknowledgments}

This work was financially supported in part by the Grant-in-Aid for Scientific Research (A) (2) (nos. 09358020 and 12308047) from the Ministry of Education, Science and Culture, Japan. The authors wish to thank Mr. Y. Ohara, their former student, for his contribution to the preparation of cells.

\section{References}

(1) Campbell, G.R. and Campbell, J.H., Smooth Muscle Phenotypic Changes in Arterial Wall Homeostasis: Implications for the Pathogenesis of Atherosclerosis, Exp. Mol. Pathol., Vol. 42 (1985), pp. 139-162.

(2) Chamley-Campbell, J., Campbell, G.R. and Ross, R., The Smooth Muscle Cell in Culture, Physiol. Rev., Vol. 59 (1979), pp. 1-61.

(3) Owens, G.K., Regulation of Differentiation of Vascular Smooth Muscle Cells, Physiol. Rev., Vol. 75 (1995), pp. 487-517.

(4) Thyberg, J., Hedin, U., Sjölund, M., Palmberg, L. and Bottger, B.A., Regulation of Differentiated Properties and Proliferation of Arterial Smooth Muscle Cells, Arteriosclerosis, Vol.10 (1990), pp. 966-990.

(5) Small, J.V. and Gimona., M., The Cytoskeleton of the Vertebrate Smooth Muscle Cell, Acta Physiol. Scand., Vol. 164 (1998), pp. 341-348.

(6) Cox, R.H., Effects of Age on the Mechanical Properties of Rat Carotid Artery, Am. J. Physiol., Vol. 233 (1977), pp. H256-H263.

( 7 ) Dobrin, P.B. and Rovick, A.A., Influence of Vascular Smooth Muscle on Contractile Mechanics and Elasticity of Arteries, Am. J. Physiol., Vol. 217 (1969), pp. 1644-1651.

(8) Desmoulière, A. and Gabbiani, G., Smooth Muscle Cell and Fibroblast Biological and Functional Features: Similarities and Differences. In: The vascular smooth muscle cell, edited by Schwartz, S.M. and Mecham, R.P., (1995), pp. 329-359, Academic Press, San Diego.

(9) Birukov, K.G., Shirinsky, V.P., Stepanova, O.V., Tkachuk, V.A., Hahn, A.W.A., Resink, T.J. and Smirnov, V.N., Stretch Affects Phenotype and Proliferation of Vascular Smooth Muscle Cells, Mol. Cell. Biochem., Vol. 144 (1995), pp. 131-139.

(10) Chamley-Campbell, J. and Campbell, G.R., What Controls Smooth Muscle Phenotype?, Atherosclerosis, Vol. 40 (1981), pp. 347-357.

(11) Reusch, P., Wagdy, H., Reusch, R., Wilson, E. and
Ives, H.E., Mechanical Strain Increases Smooth Muscle and Decreases Nonmuscle Myosin Expression in Rat Vascular Smooth Muscle Cells, Circ. Res. Vol. 79 (1996), pp. 1046-1053.

(12) Sartore, S., Chiavegato, A., Franch, R., Faggin, E. and Pauletto, P., Myosin Gene Expression and Cell Phenotypes in Vascular Smooth Muscle during Development, in Experimental Models, and in Vascular Disease, Arterioscler. Thromb. Vasc. Biol., Vol. 17 (1997), pp. 1210-1215.

(13) Williams, B., Mechanical Influences on Vascular Smooth Muscle Cell Function, J. Hypertens., Vol. 16 (1998), pp. 1921-1929.

(14) Ross, R., The Pathogenesis of Atherosclerosis: A Perspective for the 1990s, Nature, Vol. 362 (1993), pp. 801-809.

(15) Bevan, R.D., Van Marthens, E. and Bevan, J.A., Hyperplasia of Vascular Smooth Muscle in Experimental Hypertension in the Rabbit, Circ. Res., Vol. 38 (Suppl. II) (1976), pp. II-58-II-62.

(16) Cox, R.H., Alterations in Active and Passive Mechanics of Rat Carotid Artery with Experimental Hypertension, Am. J. Physiol., Vol. 237 (1979), pp. H597-H605.

(17) Sato, M., Levesque, M.J. and Nerem, R.M., Micropipette Aspiration of Cultured Bovine Aortic Endothelial Cells Exposed to Shear Stress, Arteriosclerosis, Vol. 7 (1987), pp. 276-286.

(18) Wang, N., Mechanical Interactions among Cytoskeletal Filaments, Hypertension, Vol. 32 (1998), pp. 162-165.

(19) Petersen, N.O., McConnaughey, W.B. and Elson, E.L. Dependence of Locally Measured Cellular Deformability on Position on the Cell, Temperature, and Cytochalasin B, Proc. Natl. Acad. Sci. USA, Vol. 79 (1982), pp. 5327-5331.

(20) Ting-Beall, H.P., Lee, A.S. and Hochmuth, R.M., Effect of Cytochalasin D on the Mechanical Properties and Morphology of Passive Human Neutrophils, Ann. Biomed. Eng., Vol. 23 (1995), pp. 666671.

(21) Glerum, J.J., Van Mastrigt, R. and Van Koeveringe, A.J., Mechanical Properties of Mammalian Single Smooth Muscle Cells. III. Passive Properties of Pig Detrusor and Human a Terme Uterus Cells, J. Muscle Res. Cell Motil., Vol. 11 (1990), pp. 453-462.

(22) Miyazaki, H. and Hayashi, K., Atomic Force Microscopic Measurement of the Mechanical Properties of Intact Endothelial Cells in Fresh Arteries, Med. Biol. Eng. Comput., Vol. 37 (1999), pp. 530-536.

(23) Miyazaki, H., Hasegawa, Y. and Hayashi, K., A Newly Designed Tensile Tester for Cells and Its Application to. Fibroblasts, J. Biomech., Vol. 33 (2000), pp. 97-104.

(24) Thoumine, O. and Ott, A., Time Scale Dependent Viscoelastic and Contractile Regimes in Fibroblasts Probed by Microplate Manipulation, J. Cell Sci., Vol. 110 (1997), pp. 2109-2116. 
(25) Matsumoto, T., Sato, J., Yamamoto, M. and Sato, M., Smooth Muscle Cells Freshly Isolated from Rat Thoracic Aortas Are Much Stiffer Than Cultured Bovine Cells: Possible Effect of Phenotype, JSME Int. J. Ser. C, Vol. 43, No. 4 (2000), pp. 867-874.

(26) Miyazaki, H. and Hayashi, K., Tensile Tests of Collagen Fibers Obtained from the Rabbit Patellar Tendon, Biomed. Microdevices, Vol. 2 (1999), pp. 151-157.

(27) Ross, R., The Smooth Muscle Cell. II. Growth of Smooth Muscle in Culture and Formation of Elastic Fibers, J. Cell Biol., Vol. 50 (1971), pp. 172-186.

(28) Halayko, A.J., Salari, H., Ma, X. and Stephens, N.L., Markers of Airway Smooth Muscle Cell
Phenotype, Am. J. Physiol., Vol. 270 (1996) pp. L1040-L105I.

(29) Ives, H.E., Schultz, G.S., Galardy, R.E. and Jamieson, J.D., Preparation of Functional Smooth Muscle Cells from the Rabbit Aorta, J. Exp. Med., Vol. 148 (1978), pp. 1400-1413.

(30) Gabbiani, G., Kocher, O. and Bloom, W.S., Actin Expression in Smooth Muscle Cells of Rat Aortic Intimal Thickening, Human Atheromatous Plaque, and Cultured Rat Aortic Media, J. Clin. Invest., Vol. 73 (1984), pp. 148-152.

(31) Gunst, S.J. and Tang, D.D., The Contractile Apparatus and Mechanical Properties of Airway Smooth Muscle, Eur. Respir. J., Vol. 15 (2000), pp. 600-616. 